\title{
A fermented barley and soybean formula enhances skin hydration
}

\author{
Sein Lee, ${ }^{1,2,+}$ Jong-Eun Kim, ${ }^{1,2,+}$ Sujin Suk, ${ }^{3,+}$ Oh Wook Kwon, ${ }^{4}$ Gaeun Park, ${ }^{1,2}$ Tae-gyu Lim, ${ }^{1,2}$ Sang Gwon Seo, ${ }^{1,2}$ \\ Jong Rhan Kim, ${ }^{1,2}$ Dae Eung Kim, ${ }^{5}$ Miyeong Lee, ${ }^{6}$ Dae Kyun Chung, ${ }^{6,7}$ Jong Eun Jeon, ${ }^{5}$ Dong Woon Cho, \\ Byung Serk Hurh, ${ }^{5}$ Sun Yeou Kim ${ }^{8}$ and Ki Won Lee ${ }^{1,2,9, *}$
}

\begin{abstract}
${ }^{1}$ WCU Biomodulation Major and ${ }^{3}$ Interdisciplinary Program in Agricultural Biotechnology Major, Department of Agricultural Biotechnology, ${ }^{2}$ Advanced Institutes of Convergence Technology and 9 Institute on Aging, Seoul National University, Seoul 151-921, Korea ${ }^{4}$ Graduate School of East-West Medical Science, Kyung Hee University, Global Campus, \#1732 Deogyeong-daero, Giheung-gu, Yongin, Gyeonggi-do, 446-701, Korea ${ }^{5}$ Sempio Fermentation Research Center; \#183 Osongsaengmyeong 4-ro, Osongeup, Cheongwongun, Chungcheongbukdo, 363-954, Korea ${ }^{6}$ Skin Biotechnology Center, Gyeonggi Biocenter, Suwon, Korea

${ }^{7}$ Graduate School of Biotechnology and Institute of Life Science and Resources, Kyung Hee University, Yongin 446-701, Korea

${ }^{8}$ College of Pharmacy, Gachon University, \#191 Hambakmoero, Yeonsu-gu, Incheon 406-799, Korea
\end{abstract}

Skin hydration is one of the primary aims of beauty and antiaging treatments. Barley (Hordeum vulgare) and soybean (Glycine max) are major food crops, but can also be used as ingredients for the maintenance of skin health. We developed a natural productbased skin treatment using a barley and soybean formula (BS) incorporating yeast fermentation, and evaluated its skin hydration effects as a dietary supplement in a clinical study. Participants ingested a placebo- $(n=33)$ or BS- $(3 \mathrm{~g} /$ day $)$ containing drink $(n=32)$ for 8 weeks. A significant increase in hydration in the BS group as compared to the placebo group was observed on the faces of subjects after 4 and 8 weeks, and on the forearm after 4 weeks. Decreases in stratum corneum (SC) thickness were also observed on the face and forearm. BS enhanced hyaluronan (HA) and skin barrier function in vitro and reduced $\mathrm{Hyal} 2$ expression in human dermal fibroblasts (HDF). BS also recovered ultraviolet (UV) B-induced downregulation of $\mathrm{HA}$ in $\mathrm{HaCaT}$ cells. These results suggest that BS has promising potential for development as a health functional food to enhance skin health.

Key Words: skin hydration, barley, soybean, ultraviolet B, hyaluronan

Sin is the largest organ of the human body and the critical $\checkmark$ protective barrier between our body and the environment. ${ }^{(1)}$ Skin care is considered an important priority in overall beauty care. The hydration of skin is essential for its attractive appearance, softness, complexion, protection, and helps to reinforce its barrier properties against deleterious and exogenous environmental factors..$^{(2)}$ Properly hydrated skin is smooth and soft, while inadequately hydrated skin appears dry and scaly, and exhibits poor barrier function. ${ }^{(3)}$ Consumers are generally receptive toward functional food ingredients in skincare products. ${ }^{(4)}$

Intrinsic water binding capacity and barrier function are key properties that improve with adequate skin hydration. In the extracellular matrix (ECM) of the skin, hyaluronan (HA) plays an important role in hydration due to its water-binding properties. ${ }^{(5)}$ HA is a linear, high molecular-weight polysaccharide composed of a non-sulfated repeating disaccharide unit of glucuronic acid and $\mathrm{N}$-acetylglucosamine. ${ }^{(6)} \mathrm{HA}$ exists ubiquitously as one of the major components of extracellular matrices of tissues, and effectively retains water, absorbing more than 1,000 times its weight. The HA content in skin accounts for approximately half the total amount of HA present in the body. HA is synthesized by hyaluronan synthase (HAS) at the inner surface of the plasma membrane, and the newly synthesized chains are thought to be extruded into the extracellular space through pore-like structures composed of HAS and membrane phospholipids. Three different HAS genes, HAS 1-3, have been identified in mammals. However, HA is degraded by the enzyme hyaluronidase (HYAL). HYAL-1 and HYAL-2 are major components of somatic tissue and are believed to act in concert to degrade high molecularweight HA to disaccharides and tetrasaccharides. ${ }^{(7)}$

Healthy skin is an effective barrier for maintaining water retention. ${ }^{(8)}$ Skin barrier dysfunction can be influenced by the dysregulation of factors including aquaporin-3 (AQP3), loricrin, involucrin, and filaggrin. Profilaggrin is the precursor protein of filaggrin, and is composed of 2 to 3 repeating filaggrin monomers. ${ }^{(8)}$ Profilaggrin initially decomposes to filaggrin, which is then degraded into free amino acids (FAA) which are a major component of natural moisturizing factors. ${ }^{(9)}$ AQP3 represents a family of water channel proteins which are found in the basal cell layer of the epidermis, and are essential transporters of glycerol and water. ${ }^{(10)}$ Ultraviolet (UV) light is one of major causes of damage to the skin barrier and induces detectable changes in the expression of these skin barrier markers. Proper ongoing maintenance of appropriate levels of epidermal hydration helps to mitigate the skin aging process.

Soybeans (Glycine max) are an important plant protein source worldwide, harboring various nutrients and functional components including isoflavonoids. ${ }^{(1)}$ Isoflavone in particular has been reported to exhibit bioactive properties including preventive effects toward cancers and menopausal symptoms, as well as antiinflammatory and anti-cardiovascular disease properties. ${ }^{(12)} \mathrm{Simi}-$ larly, barley (Hordeum vulgare) is a major food crop that is used primarily as a cereal, but also as an industrial commodity in fermented products. $\beta$-glucan, an active constituent of barley, has been reported to prevent skin problems. ${ }^{(13)}$ In a previous study, we developed a fermented barley and soybean formula (BS) with enhanced isoflavone (genistein and daidzein) and $\beta$-glucan content by yeast fermentation. BS provided protection against ultraviolet (UV) B-induced photoaging effects in hairless mice. ${ }^{(14)}$ In this study, we investigated the skin hydration effects of BS in a clinical trial and assessed changes in molecular markers of skin hydration in vitro.

These authors contributed equally to this work *To whom correspondence should be addressed. E-mail: kiwon@snu.ac.kr 


\section{Materials and Methods}

Chemicals and reagents. BS was prepared as described previously. ${ }^{(14)}$ Primary human dermal fibroblasts (HDFs) were isolated from the outgrowth of foreskin obtained from 7-30-yearold healthy volunteers by JH Chung's laboratory (Seoul National University Hospital, Korea) after approval from the Institutional Review Board at Seoul National University Hospital and Seoul

Table 1. Drink preparation for clinical trial

\begin{tabular}{lcccc}
\hline \multirow{2}{*}{ Materials } & \multicolumn{2}{c}{ Placebo group } & \multicolumn{2}{c}{ Experimental group } \\
\cline { 2 - 5 } & Mixing Ratio (\%) & Amount per day (g) & Mixing Ratio (\%) & Amount per day (g) \\
\hline BS & 0 & 0 & 3 & 3 \\
Water & 95.24 & 95.24 & 92.24 & 92.24 \\
Roasted unpolished rice extract & 1 & 1 & 1 & 1 \\
Fructooligosaccarides & 2 & 2 & 2 & 2 \\
Cereal flavor & 1 & 1 & 1 & 1 \\
Lotus leaf extract & 0.3 & 0.3 & 0.3 & 0.3 \\
Rice flavor & 0.2 & 0.2 & 0.2 & 0.2 \\
Enzyme treated stevia & 0.06 & 0.06 & 0.06 & 0.06 \\
Pectin & 0.1 & 0.1 & 0.1 & 0.1 \\
Dextrin & 0.1 & 0.1 & 0.1 & 0.1 \\
\hline Sum & 100 & 100 & 100 & 100 \\
\hline
\end{tabular}

A
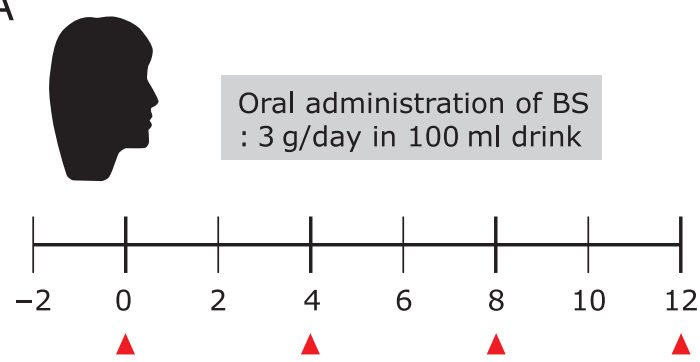

$\Delta$ : Measure skin hydration of front cheek and interior of forearm

Oral administration of BS

: $3 \mathrm{~g} /$ day in $100 \mathrm{ml}$ drink

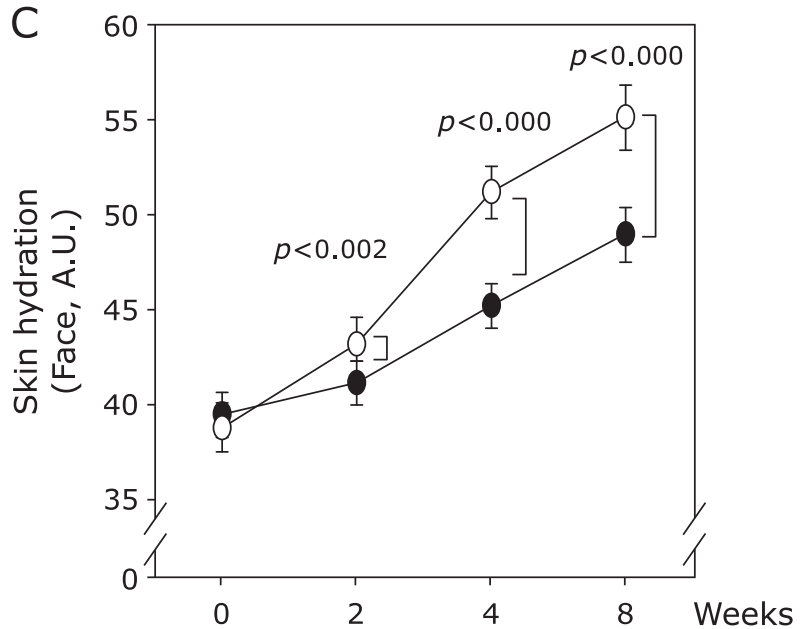

B
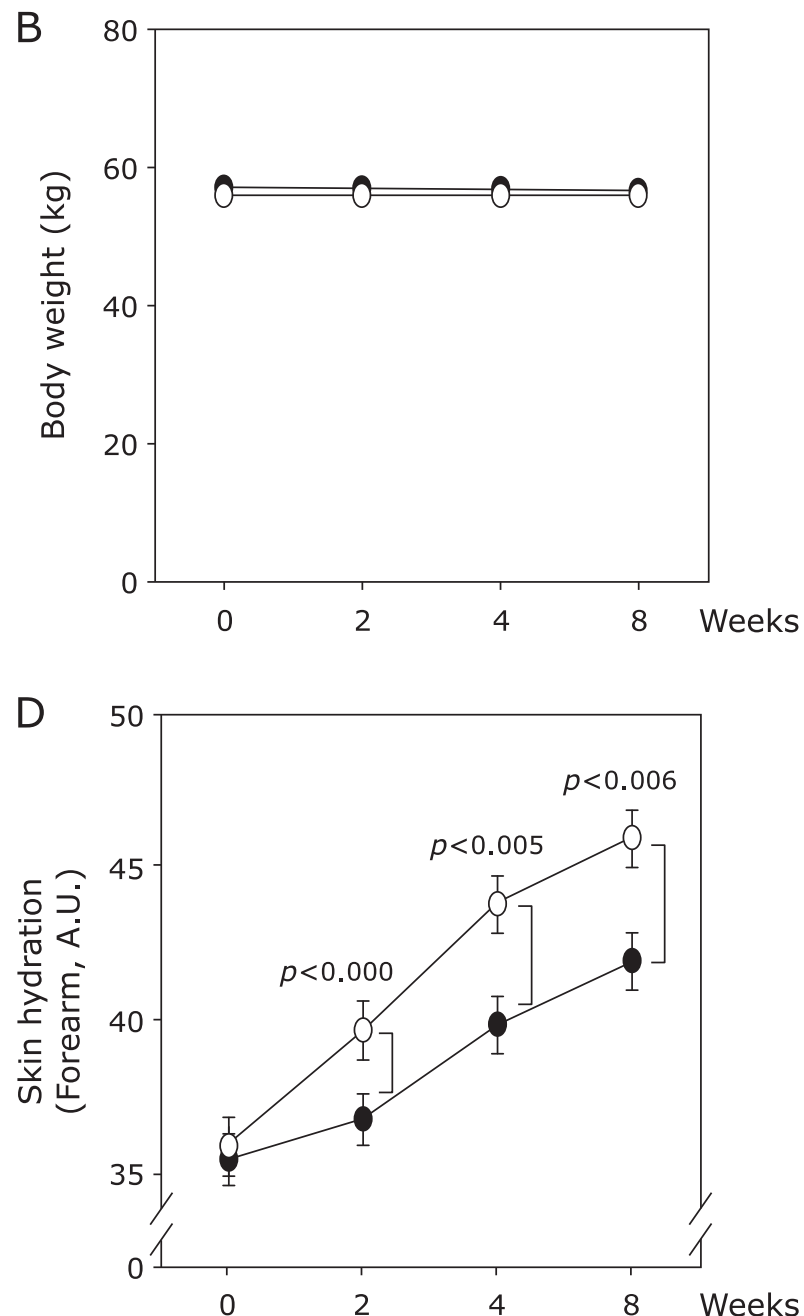

Fig. 1. Fermented barley and soybean formula (BS) enhances skin hydration in healthy subjects. (A) Clinical study design. (B) Body weight did not change significantly as a result of BS treatment. (C) and (D) BS enhances skin hydration on the face (C) and forearm (D). The clinical study was conducted as described in Materials and Methods. Skin hydration was measured using a Corneometer ${ }^{\circledR}$ CM825. Open circle $=$ placebo group, closed circle $=$ BS group. Data represent mean values \pm SD. $P$ values were determined with student's $t$ test. 
A
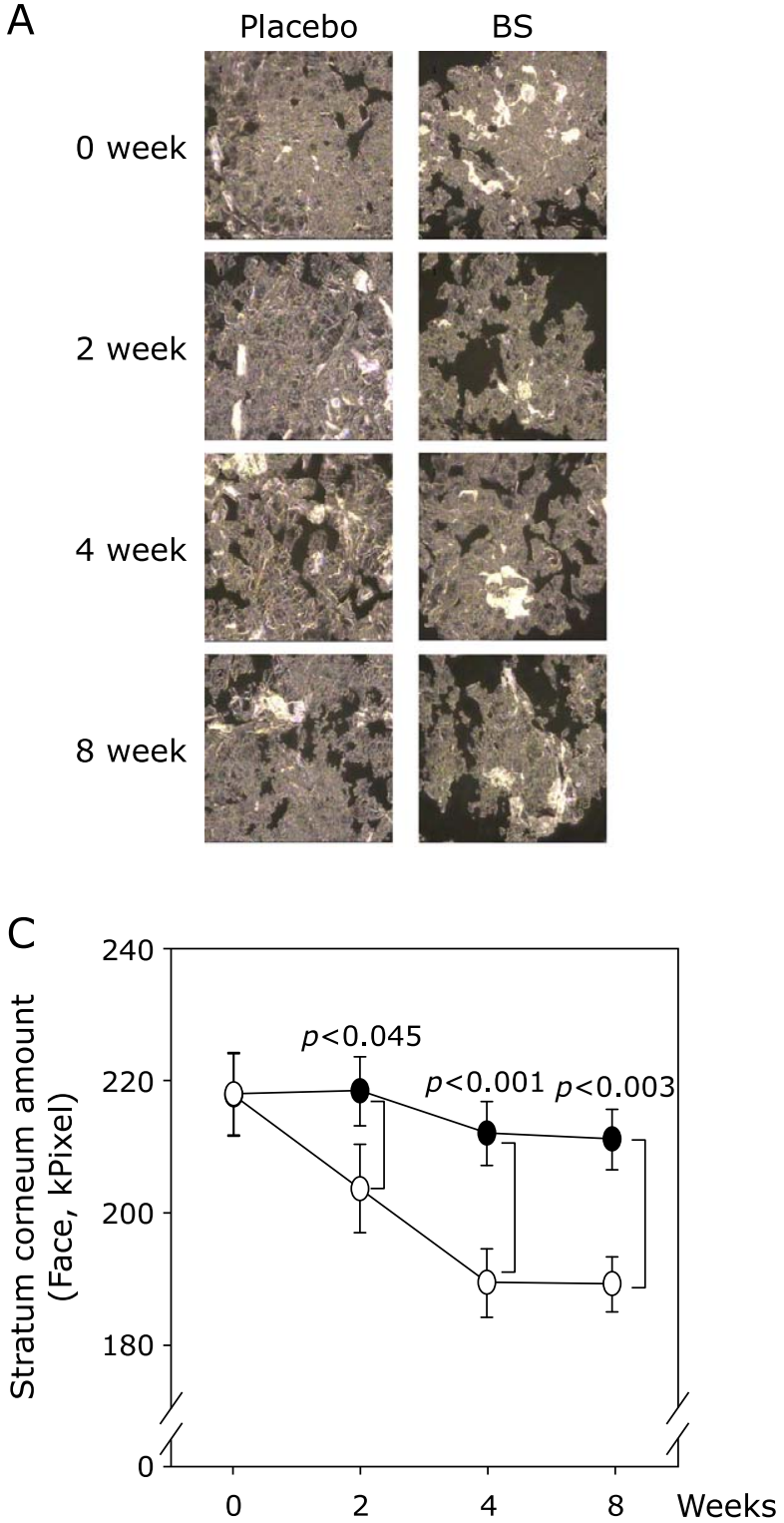

B
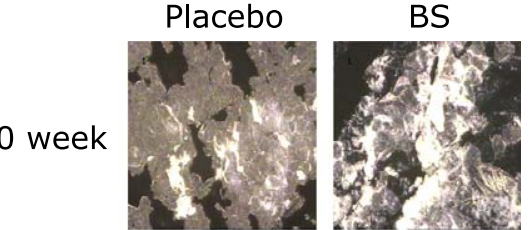

2 week
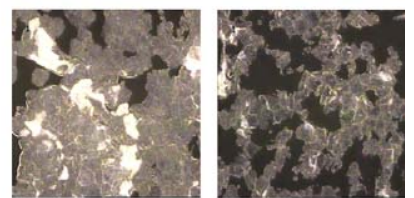

4 week
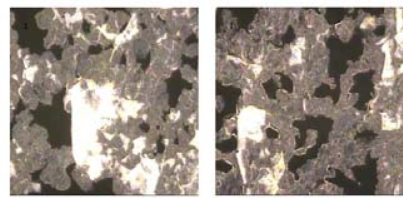

8 week
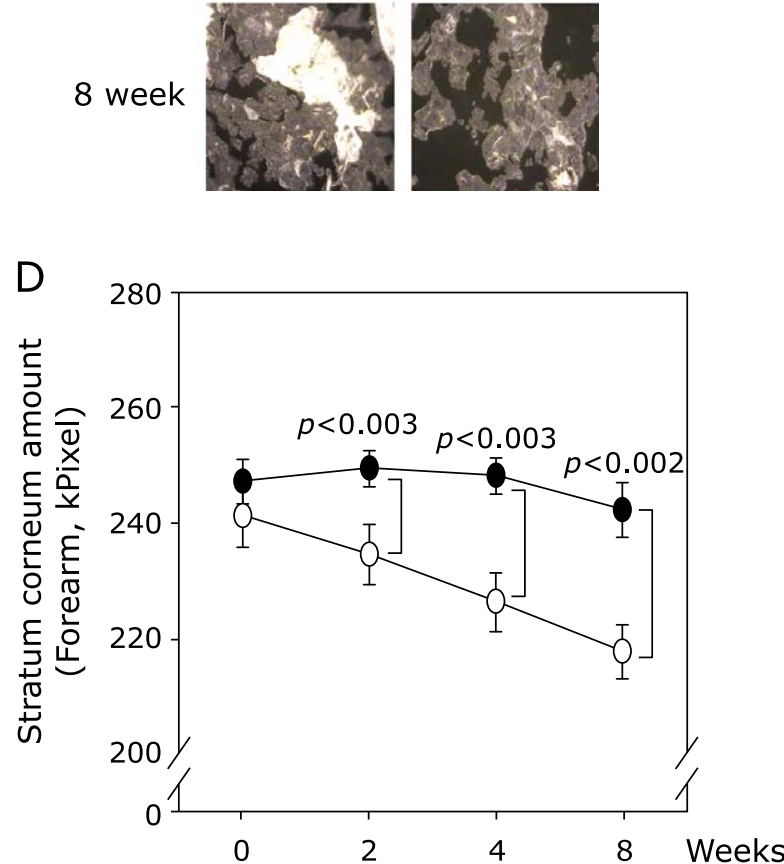

Fig. 2. BS reduces stratum corneum thickness in healthy subjects. (A) and (B) Stratum corneum images of placebo and BS-treated subjects taken at the indicated time points. Quantification of stratum corneum using D-squame ${ }^{\oplus}$ Black Tape on the face (A) and forearm (B). (C) and (D) quantification of the stratum corneum in face (C) and forearm (D) images using Image-Pro ${ }^{\oplus}$ Plus software (Media Cybernetics Inc, MD). The open circle represents the placebo group and closed circle represents the BS group. Data represent mean values \pm SD. $P$ values were determined using student's $t$ test.

National University. HaCaT cells were purchased from CLS Cell Lines Service GmbH (Eppelheim, Germany). Dulbecco's modified eagle medium (DMEM) was purchased from Hyclone (Long, UT). Fetal bovine serum (FBS) and $\beta$-actin antibody were bought from Sigma-Aldrich (St. Louis, MO). The AQP-3, $\alpha$ tubulin, Hyal2 and HAS2 antibodies was obtained from Santa Cruz Biotechnology (Santa Cruz, CA). Antibodies against filaggrin were purchased from Millipore (Billerica, MA). 3-(4,5dimethylatiazol-2-yl)-2,5 diphenyltetrazolium bromide (MTT) powder was purchased from USB Co. (Cleveland, OH). Penicillin/ streptomycin was purchased from Invitrogen (Grand Island, NY). Protein assay kits were obtained from Bio-Rad Laboratories (Hercules, CA).

Clinical study with healthy volunteers. Sixty five healthy volunteers aged 25 to 60 years with dry and dark skin were recruited for the clinical study (Supplemental Table 1*). The Medical Ethics Committee of Kyung Hee University approved the trial (KHUSBC 2014-001). The participants were randomly assigned to two groups [control $(n=33)$ and experimental group $(n=32)]$, and then randomly assigned to two subgroups within each group to examine each side of the face. Participants were given a placebo or BS-containing drink $(100 \mathrm{ml}$, Table 1$)$ for 8 weeks. Participants were banned from using certain functional cosmetics, external applicators, and dietary supplements from two weeks before the study until its conclusion. Hydration under the dead skin (10-20 $\mu \mathrm{m}$ of the stratum corneum) was examined using a Corneometer ${ }^{\circledR}$ CM825 according to the manufacturer's instructions (Courage + Khazaka Electronic GmbH, Cologne, Germany). The amount of stratum corneum in the interior of the forearm and front cheek were examined using D-squame ${ }^{\mathbb{B}}$ Black Tape. Images of the collected dead skin samples were magnified 70X using Charm View (Moritex, Japan) and analyzed with Image-Pro ${ }^{\circledR}$ Plus software (Media Cybernetics Inc, MD). 
A

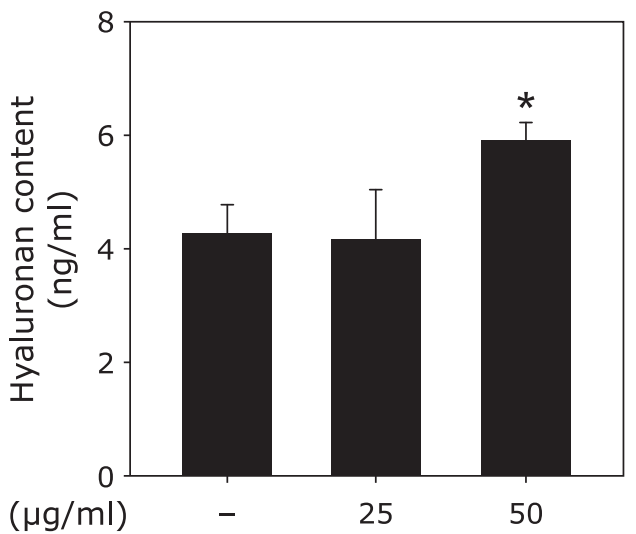

C

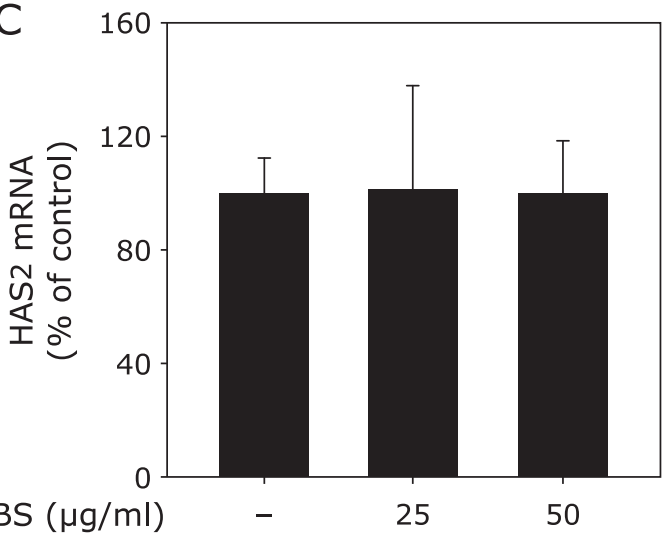

E

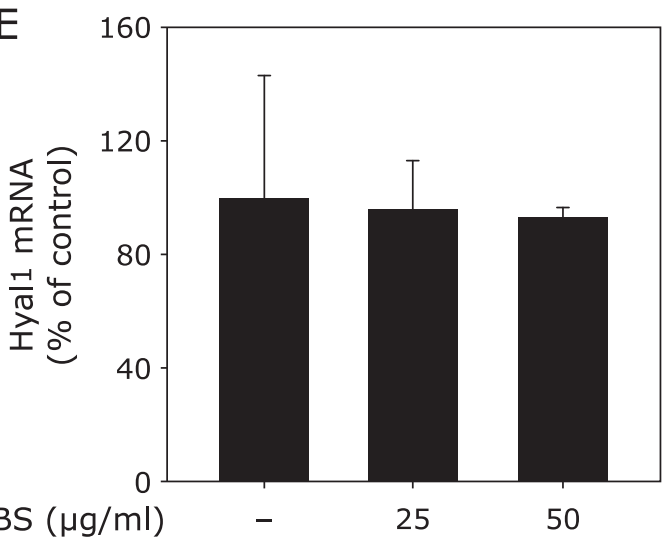

B

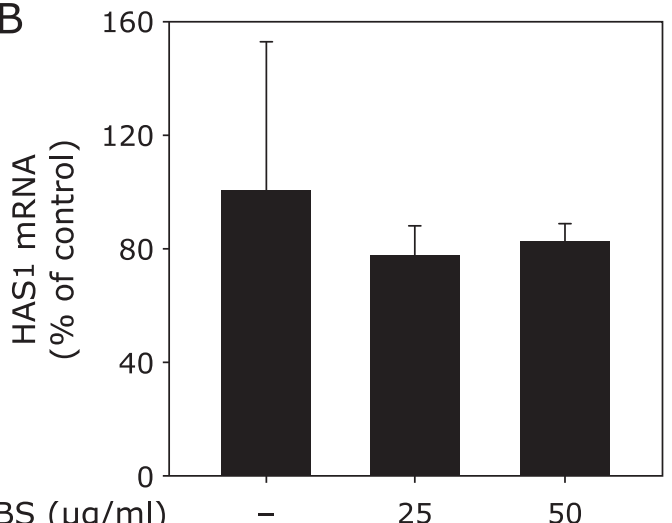

BS $(\mu \mathrm{g} / \mathrm{ml}) \quad-\quad 25 \quad 50$

D

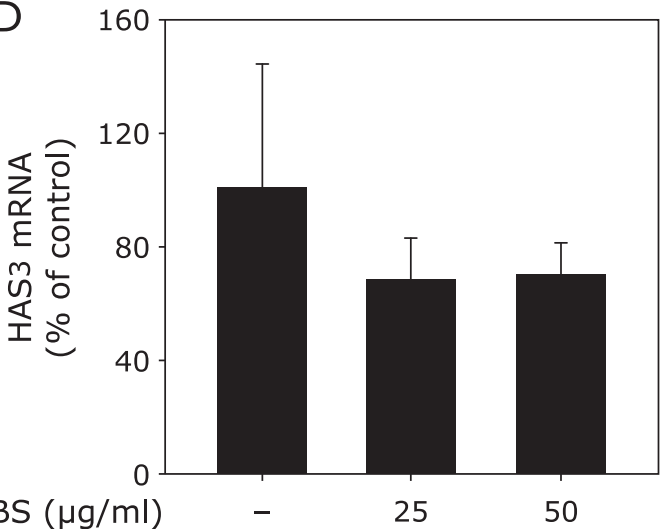

$\mathrm{F}$

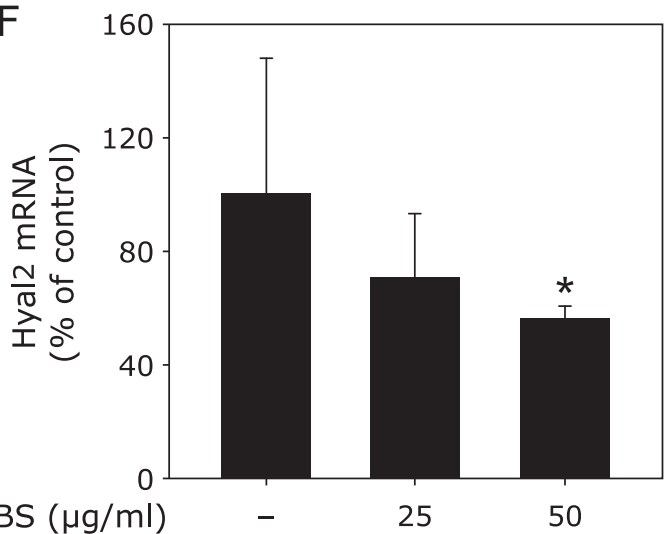

G

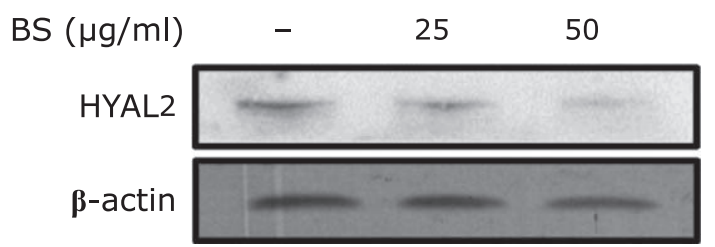

Fig. 3. BS enhances hyaluronan (HA) content via inhibition of HYAL2 in human dermal fibroblasts (HDF). (A) BS enhances HA content in HDFs. HDF cells were starved in serum-free media and treated with BS at the indicated concentrations for $48 \mathrm{~h}$. HA concentrations were determined by ELISA as described in Materials and Methods. (B), (C), (D), (E) and (F) effects of BS on mRNA expression of an HA-regulatory enzyme in HDF cells. HDF cells were starved in serum-free media and treated with BS at the indicated concentrations for $24 \mathrm{~h}$. mRNA levels of HAS1 (B), HAS2 (C), HAS3, HYAL1 (E) and HYAL2 (F) were analyzed by real-time RT-PCR using specific primers as described in Materials and Methods. (G) HA reduces HYAL2 protein expression in HDF cells. HDF cells were starved in serum-free media and treated with BS at the indicated concentrations for $48 \mathrm{~h}$. HYAL2 and $\beta$-actin expression were determined by Western blot analysis as described in Materials and Methods. Data represent mean values \pm SD. ${ }^{\star} p<0.05$ vs the nontreated control. 
UVB irradiation. UVB irradiation was conducted with cells in serum-free media. The spectral peak of the UVB (Bio-Link crosslinker, VilberLourmat, Cedex 1, France) was set at $312 \mathrm{~nm}$. $\mathrm{HaCaT}$ cells were exposed to UVB at $0.01 \mathrm{~J} / \mathrm{cm}^{2}$.

Real time RT-PCR. Cells were harvested in RNAiso Plus (Takara Bio, Inc., Shiga, Japan). After reverse transcription with oligo-dT primers using PrimeScriptTM 1st strand cDNA synthesis Kit (Takara Bio, Inc.), the cDNA was probed with the following primers (Bioneer, Daejeon, Korea): HAS1 Fw: 5'-GGAATAACCTCTTGCAGCAGTTTC-3' Rv: 5'-GCCGGTCATCCCCAAAAG-3' HAS2 Fw: 5'-TCGCAACACGTAACGCAAT-3', Rv: 5'-ACTTCTCTTTTTCCACCCCATTT-3', HAS3 Fw: 5'AACAAGTACGACTCATGGATTTCCT-3'，Rv: 5'-GCCCGCTCCACGTTGA-3', HYAL1 Fw: 5'-GATGTCAGTGTCTTCGATGTGGTA-3'，Rv: 5'-GGGAGCTATAGAAAATTGTCATGTCA-3', HYAL2 Fw: 5'-CTAATGAGGGTTTTGTGAACCAGAATAT-3', Rv: 5'-GCAGAATCGAAGCGTGGATAC-3' PCR was performed using a Gene Amp PCR System 2700 (Applied Biosystems, Foster City, CA). Real time-PCR was performed with a CFX96 real-time PCR detection system (Bio-Rad, Hercules, CA). cDNA was amplified in the presence of $\mathrm{iQ}^{\mathrm{TM}}$ SYBR $^{\circledR}$ Green Supermix (Bio-Rad). To control variations in mRNA concentration, all results were normalized to the housekeeping gene, GAPDH. Relative quantitation was performed using a comparative $\Delta \Delta \mathrm{Ct}$ method following the manufacturer's instructions.

Enzyme linked immunosorbent assay (ELISA). Conditioned media was collected after $48 \mathrm{~h}$ of incubation. The samples were measured using a DuoSet human total Hyaluronan ELISA kit (R\&D Systems Inc.) as described in the manufacturer's protocol.

Western blot assay. The cells were scraped with lysis buffer [10 mM Tris ( $\mathrm{pH} 7.5), 150 \mathrm{mM} \mathrm{NaCl}, 5 \mathrm{mM}$ ethylene diamine tetra acetic acid (EDTA), 1\% Triton X-100, $1 \mathrm{mM}$ dithiothreitol (DTT), $0.1 \mathrm{mM}$ phenylmethylsulfonyl fluoride (PMSF), $10 \%$ glycerol and protease inhibitor cocktail tablet] on ice for $20 \mathrm{~min}$, and then centrifuged at $18,620 \times g$ for $10 \mathrm{~min}$. The protein concentration was measured with a dye-binding protein assay kit (BioRad Laboratories) as described by the manufacturer. The proteins were separated electrophoretically in $10 \%$ SDS-polyacrylamide gel and transferred to an Immobilon $\mathrm{P}$ membrane (EMD Millipore). The membrane was blocked with 5\% fat-free milk for $1 \mathrm{~h}$ and then incubated with the specific primary antibody at $4^{\circ} \mathrm{C}$ overnight. Protein bands were visualized with a chemiluminescence detection kit (GE Healthcare, NJ) after hybridization with an HRP-conjugated secondary antibody (Santa Cruz Biotechnology) or IRDye ${ }^{\circledR}$ IRDye $^{\circledR} 700 \mathrm{DX}$ conjugated secondary antibodies (Lincoln, NE).

Clinical study with healthy volunteers. Sixty five healthy volunteers aged 25 to 60 years with dry and dark skin were recruited for the clinical trial (Supplemental Table 1*). The Medical Ethics Committee of Kyung Hee University approved the trial (KHUSBC 2014-001). Participants were randomly assigned to two groups [control $(n=33)$ and experimental group $(n=32)$ ], and then randomly assigned to two subgroups groups within each group to examine each side of the face. Participants took placebo drink or BS containing drink $(100 \mathrm{ml}$, Table 1$)$ for 8 weeks. Participants were banned from using certain functional cosmetics, external application, and dietary supplement from two weeks before study to the end of the study. Hydration under the dead skin (10-20 $\mu \mathrm{m}$ of the Stratum corneum) was examined using the Corneometer $^{\circledR}$ CM825 according to the manufacturer's instructions (Courage + Khazaka Electronic GmbH, Cologne, Germany). Amount of stratum corneum in the interior of the forearm and front cheek were examined with D-squame ${ }^{\circledR}$ Black Tape. The image of the collected dead skin samples was magnified 70 times using Charm View (Moritex, Japan) and analyzed with ImagePro $^{\circledR}$ Plus software (Media cybernetics Inc, MD).

Statistical analysis. Statistical analyses were performed using student's $t$ test. $P$ values of less than 0.05 were considered statistically significant.

\section{Results}

BS enhances skin hydration in healthy subjects. To verify the effects of BS on skin hydration, we conducted a clinical study in healthy subjects. The trial was conducted as described in Fig. 1A. Skin hydration of the front cheek and interior of the forearm of the volunteers was examined prior to initiation of the study and 2, 4 and 8 weeks after the administration of a drink containing BS ( $3 \mathrm{~g}$ added to a $100 \mathrm{ml}$ drink/day). There were no significant changes in weight (Fig. 1B) or side effects observed during the trial. Compared with the placebo group, the BS-treated group showed enhanced hydration of skin on the face (Fig. 1C) and forearm (Fig. 1D). We then assessed the stratum corneum, a marker of skin hydration. BS administration reduced the quantity of stratum corneum present on the face (Fig. 2A and C) and forearm (Fig. 2B and D). These results suggest that BS has a positive effect on skin hydration in humans.

BS upregulates HA via inhibition of Hyal2 expression in HDFs. HA is a major humectant in the skin. ${ }^{(5)}$ The viscoelastic properties of HA and its capacity for hydration are largely dependent on the molecular weight of HA. ${ }^{(6)}$ During periods of rapid growth and tissue remodeling, HA fills the vacant spaces created within the skin and undergoes deformation to maintain tissue hydration and buffer the local environment. To validate effects on skin hydration, we treated HDFs with the BS formula. Treatment with BS was observed to increase HA levels in the skin fibroblasts (Fig. 3A). HA is synthesized by HAS1, HAS2, and HAS3, and is degraded by Hyall and Hyal2. There were no significant changes in mRNA levels of HAS1, HAS2, HAS3 or Hyal1, although Hyal2 expression did change after BS treatment of HDFs for $24 \mathrm{~h}$ (Fig. 3B, C, D and E). Only Hyal2 mRNA was decreased by BS treatment (Fig. 3F). Protein levels of Hyal2 also decreased after BS treatment in HDFs. We therefore hypothesize that the increases in HA elicited by BS are mediated by suppression of Hyal2 expression.

BS recovers UVB-induced down-regulation of a barrier function-related gene. Healthy skin acts as a barrier to protect the body from harmful external elements while preserving internal homeostasis. ${ }^{(3)}$ Skin barrier proteins including filaggrin, loricrin

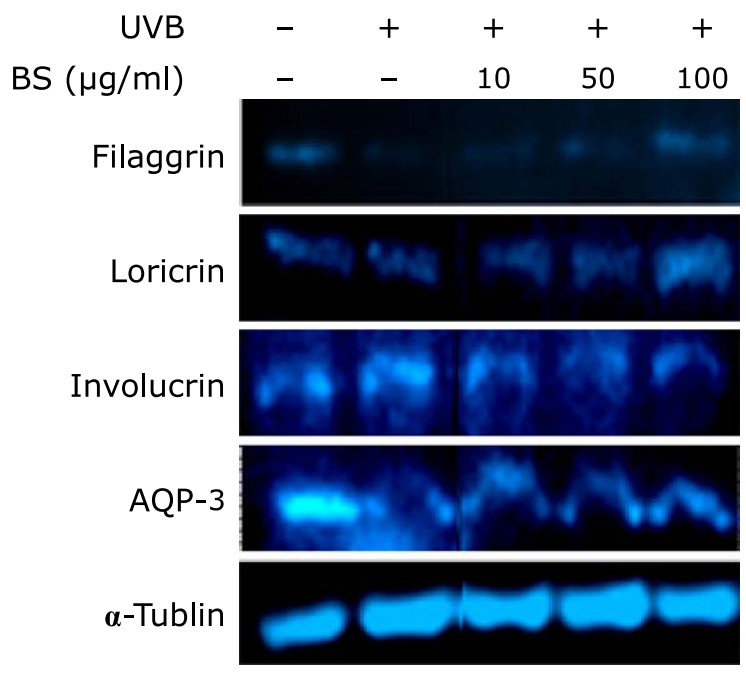

Fig. 4. BS recovers ultraviolet (UV) B-induced downregulation of a barrier function related gene in $\mathrm{HaCaT}$ cells. After $1 \mathrm{~h}$ of BS treatment, cells were irradiated with $0.01 \mathrm{~J} / \mathrm{cm}^{2}$ UVB. Protein was harvested after $24 \mathrm{~h}$ and detected with specific antibodies by Western blot as described in Materials and Methods. 
A

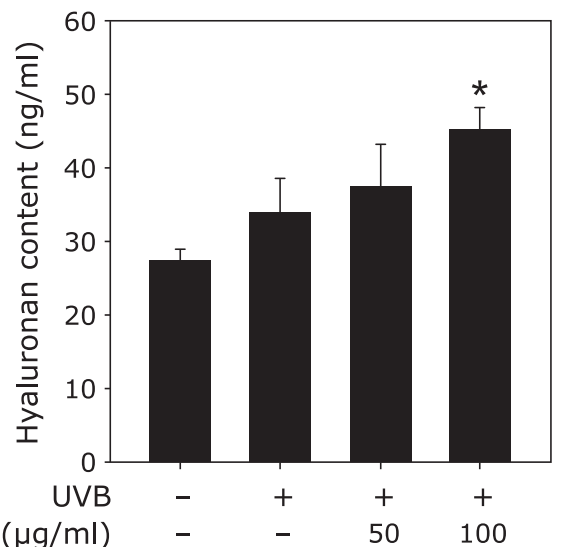

C

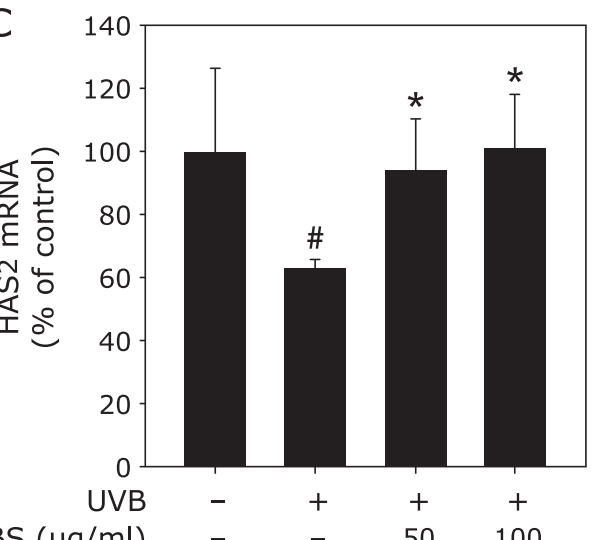

BS $(\mu \mathrm{g} / \mathrm{ml}) \quad-\quad-\quad 50100$

E

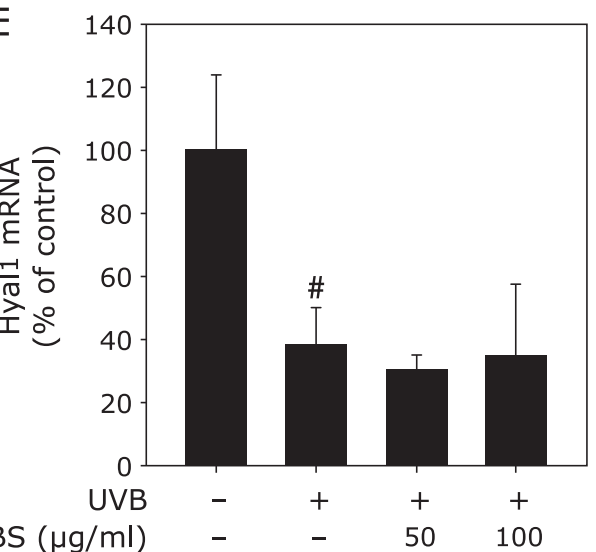

B

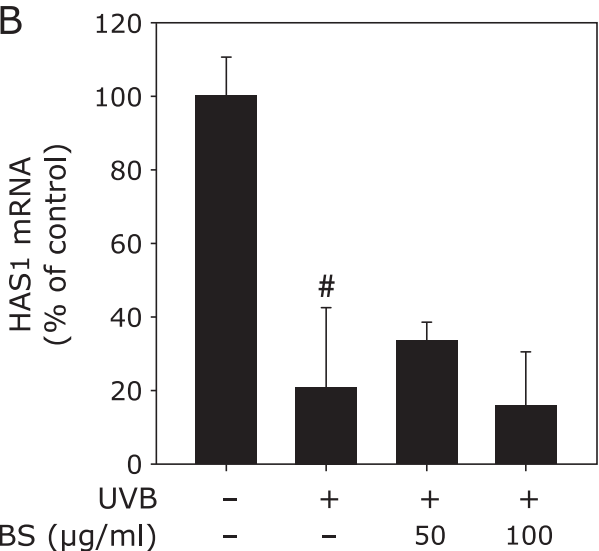

D

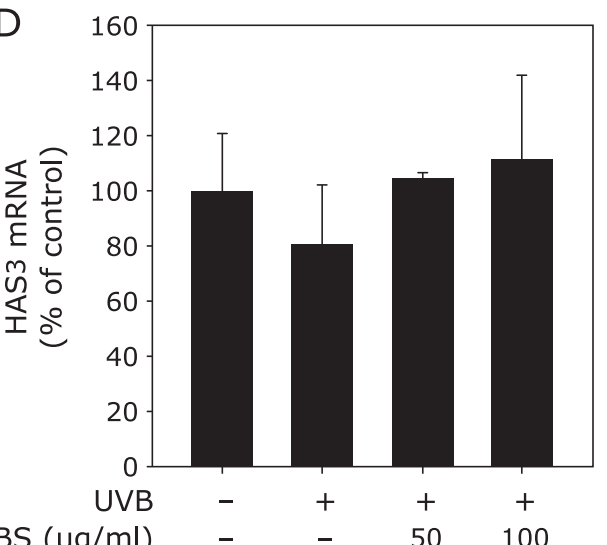

$\mathrm{F}$

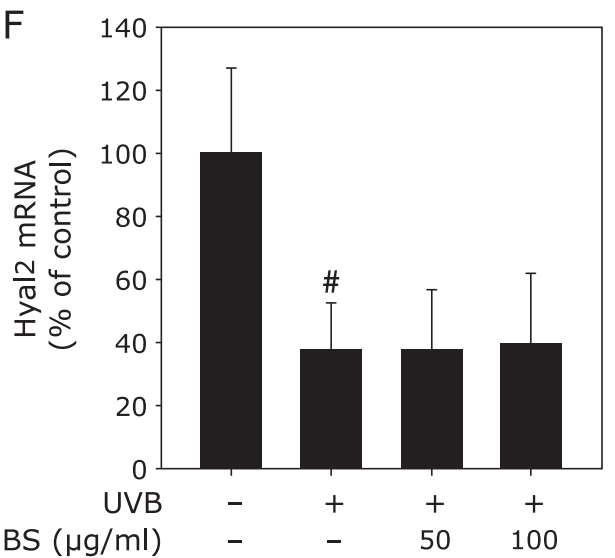

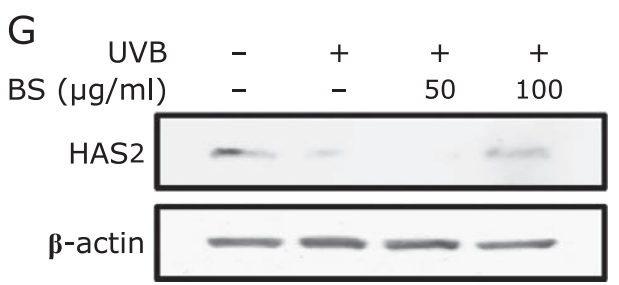

Fig. 5. BS enhances HA expression via recovery of HAS2 protein in UVB-treated HaCaT cells. (A) BS upregulates HA content in UVB-treated HaCaT cells. After $1 \mathrm{~h}$ of BS treatment, cells were irradiated with $0.01 \mathrm{~J} / \mathrm{cm}^{2}$ UVB. Media was collected after $48 \mathrm{~h}$. HA concentrations were determined by ELISA as described in Materials and Methods. (B), (C), (D), (E) and (F) effects of BS treatment on mRNA expression of an HA regulatory enzyme in UVB-treated HaCaT cells. mRNA was harvested after $48 \mathrm{~h}$. mRNA levels of HAS1 (B), HAS2 (C), HAS3, HYAL1 (E) and HYAL2 (F) were analyzed by realtime RT-PCR using specific primers as described in Materials and Methods. (G) HA reduces HYAL2 protein expression in HaCaT cells. HaCaT cells were starved in serum-free media and treated with BS at the indicated concentrations for $48 \mathrm{~h}$. HAS2 and $\beta$-actin expression were determined by Western blot as described in Materials and Methods. Data represent mean values \pm SD. ${ }^{*} p<0.01$ vs the UVB-treated control; ${ }^{\#} p<0.01$ vs the non-treated control. 
and involucrin are produced by keratinocytes and play crucial roles in skin barrier function. ${ }^{(8)}$ AQP3 downregulation in the skin leads to dehydration. ${ }^{(15)}$ We tested BS treatment on filaggrin, loricrin, involucrin and AQP3 levels following UVB irradiation in $\mathrm{HaCaT}$ cells (Fig. 4). UVB downregulated the expression of filaggrin, loricrin and AQP3, while BS treatment recovered these levels. However, involucrin levels remained unchanged by both UVB and BS treatment.

BS increases HA content via increase of HAS2 expression in UVB induced HaCaT cells. UV is a major cause of skin aging and dryness. ${ }^{(16)}$ UVB damages or downregulates a number of important biological factors in the skin, particularly in keratinocytes which exist on the surface of the skin and are easily irradiated. We tested the effect of BS on HA levels after irradiation with UV. ${ }^{(7)}$ UVB did not significantly alter HA levels. However, BS treatment was observed to upregulate HA (Fig. 5A). We measured the mRNA levels of several HA-related proteins and found that UVB inhibited HAS1, HAS2, HYAL1 and HYAL2 mRNA expression (Fig. 5B, C, D, E and F). Only HAS2 mRNA was recovered by BS treatment after UVB irradiation (Fig. 5C). The protein levels of HAS2 also followed a similar tendency with its mRNA levels (Fig. 5G).

\section{Discussion}

Adequate hydration is vital for skin health, as well-hydrated skin appears healthy and youthful. The observable link among moisturized skin, healthy skin and 'good looking' skin indicates that there are likely appearance benefits for maintaining levels of skin hydration. Consequently, skin moisturizers represent a major product category in the skincare business. ${ }^{(3)}$ In this study, we used a natural product-based treatment for skin hydration consisting of a fermented soybean and barely mixture and verified its effects on skin hydration in human volunteers. Both soybean and barley have been used as staple food sources for much of recorded history and both can be processed by yeast fermentation. ${ }^{(12,17)}$ No abnormalities in the skin, whole body or blood of the subjects were detected in the experimental period, suggesting that BS is an effective and safe natural material for skin health.

Fermentation has been developed by humanity over many centuries for the preservation of various foods. ${ }^{(18)}$ The bioconversion of components in food by bacteria can also alter the bioactive properties of the constituents. ${ }^{(19)}$ A number of recently-developed biotechnology methods may be useful for enhancing components that are beneficial for human health. ${ }^{(20)}$ We observed enhanced concentrations of daidzein, genistein, and $\beta$-glucans in barley and soybean after yeast fermentation. Daidzein and genistein exert beneficial effects on aged skin by increasing levels of collagen and hyaluronan. They also reduce matrix metalloproteinase (MMP) expression, and stimulate fibroblast proliferation.

Barley (Hordeum vulgare L.) is a major food crop, and is used primarily as a cereal but also as an industrial commodity for the production of fermented commodities. $\beta$-glucans are the active component of barley ${ }^{(21)}$ and exert beneficial effects on skin health, by decreasing hyaluronidase and collagenase expression. They also stimulate collagen protein expression, and reduce wrinkling both in vivo and in human subjects. ${ }^{(13)} \mathrm{BS}$ is itself a yeast fermentation product with enhanced daidzein, genistein, and $\beta$-glucan content. ${ }^{(14)}$

Proper skin hydration is dependent on natural hygroscopic

\section{References}

1 Watt FM. Mammalian skin cell biology: at the interface between laboratory and clinic. Science 2014; 346: 937-940.

2 Draelos ZD. Modern moisturizer myths, misconceptions, and truths. Cutis 2013; 91: 308-314.

3 Verdier-Sévrain S, Bonté F. Skin hydration: a review on its molecular agents including $\mathrm{HA},{ }^{(3)}$ which is produced by HAS and destroyed by HYAL. HAS produces HA by alternately incorporating two UDP-sugar substrates, UDP- $N$-acetylglucosamine and UDPglucuronic acid in the cytoplasm. HA is then secreted via an $\mathrm{ABC}$ transporter system. HYAL1 can digest HA into tetrasaccharides while HYAL2 cleaves high molecular weight HA into disaccharide units. ${ }^{(22)}$ We observed that BS increased HA levels in HDF cells, but did not alter HAS1, HAS2, HAS3 or HYAL1 mRNA expression. However, BS did appear to inhibit HYAL2 mRNA and protein expression levels.

Another important reason to maintain skin hydration is to improve the barrier function of the skin. ${ }^{(8)}$ Loss of water from the skin is carefully regulated by the epidermis. ${ }^{(3)}$ The proteins which play crucial roles in skin barrier function such as filaggrin, loricrin, involucrin and AQP3 are also important for skin hydration. ${ }^{(8)}$ Filaggrin monomers are derived by proteolysis from the precursor protein profilaggrin, and are then degraded into free amino acids that are major components of natural moisturizing factors. ${ }^{(9)}$ Loricrin is a major reinforcing factor for the cornified envelope. Involucrin consists of repeating peptide units and is a component of the cornified envelope, ${ }^{(23)}$ while AQP3 is found in the basal cell layer of the epidermis and is an essential transporter of glycerol and water. ${ }^{(10)}$ UVB damages the skin barrier and causes water loss via changes to the expression levels of these proteins. ${ }^{(3)}$ In this study, we observed that UVB reduced filaggrin, loricrin, and AQP3 expression, while BS rescued their expression. Involucrin levels remained unchanged by both UVB and BS treatment. Previous studies have shown that HA is slightly upregulated by UVB treatment as a defense mechanism. ${ }^{(7,24)}$ HA-regulating enzymes change in expression after irradiation with UVB. ${ }^{(25-27)}$ With our experimental conditions, mRNA levels of HAS1, HAS2, HYAL1, and HYAL2 were decreased after UVB treatment, while mRNA levels of HAS3 did not change. Only HAS2 levels were found to be recovered by BS after UVB irradiation. The HAS2 protein levels also increased, similar to the mRNA. Therefore, we hypothesize that the increase in HA content after UVB irradiation stems from recovery of HAS2 levels by BS. BS appears to enhance skin barrier function and HA content, and may help to keep water within damaged skin.

Our results demonstrate the skin hydration effects of BS in human subjects. Our in vitro mechanism studies revealed that BS upregulates HA content via inhibition of HYAL2. In conclusion, $\mathrm{BS}$ has promising potential as a natural product-based material with beneficial effects for skin health.

\section{Acknowledgments}

This work was supported by the Korea Institute of Planning and Evaluation for Technology in Food, Agriculture, Forestry and Fisheries (iPET, 311035-03-3-HD110), the R\&D program of MOTIE/KIAT (Establishment of Infra Structure for Anti-aging Industry Support, N0000697), and the Leap Research Program Grant of the National Research Foundation funded by the Ministry of Science, ICT and Future Planning (2010-0029233) in Republic of Korea.

\section{Conflict of Interest}

No potential conflicts of interest were disclosed.

\footnotetext{
Clin Croat 2010; 49: 537-544.

5 Wiest L, Kerscher M. Native hyaluronic acid in dermatology--results of an expert meeting. J Dtsch Dermatol Ges 2008; 6: 176-180.
}

. 
6 Robert L. Hyaluronan, a truly "youthful” polysaccharide. Its medical applications. Pathol Biol (Paris) 2014; 63: 32-34.

7 Kurdykowski S, Mine S, Bardey V, et al. Ultraviolet-B irradiation induces differential regulations of hyaluronidase expression and activity in normal human keratinocytes. Photochem Photobiol 2011; 87: 1105-1112.

8 Rawlings AV. Molecular basis for stratum corneum maturation and moisturization. Br J Dermatol 2014; 171 Suppl 3: 19-28.

9 McGrath JA, Uitto J. The filaggrin story: novel insights into skin-barrier function and disease. Trends Mol Med 2008; 14: 20-27.

10 Hara-Chikuma M, Verkman AS. Roles of aquaporin-3 in the epidermis. $J$ Invest Dermatol 2008; 128: 2145-2151.

11 Izumi T, Saito M, Obata A, Arii M, Yamaguchi H, Matsuyama A. Oral intake of soy isoflavone aglycone improves the aged skin of adult women. $J$ Nutr Sci Vitaminol (Tokyo) 2007; 53: 57-62.

12 Ravindranath MH, Muthugounder S, Presser N, Viswanathan S. Anticancer therapeutic potential of soy isoflavone, genistein. Adv Exp Med Biol 2004; 546: $121-165$.

13 Du B, Bian Z, Xu B. Skin health promotion effects of natural beta-glucan derived from cereals and microorganisms: a review. Phytother Res 2014; 28: 159-166.

14 Kwon OW, Venkatesan R, Do MH, et al. Dietary supplementation with a fermented barley and soybean mixture attenuates UVB-induced skin aging and dehydration in hairless mouse skin. Food Sci Biotechnol 2015; 24: 705715.

15 Ji C, Yang Y, Yang B, et al. Trans-Zeatin attenuates ultraviolet induced down-regulation of aquaporin-3 in cultured human skin keratinocytes. Int $J$ Mol Med 2010; 26: 257-263.

16 Kaur CD, Saraf S. In vitro sun protection factor determination of herbal oils used in cosmetics. Pharmacognosy Res 2010; 2: 22-25.
17 Ames NP, Rhymer CR. Issues surrounding health claims for barley. J Nutr 2008; 138: $1237 \mathrm{~s}-1243 \mathrm{~s}$.

18 Kim NY, Song EJ, Kwon DY, Kim HP, Heo MY. Antioxidant and antigenotoxic activities of Korean fermented soybean. Food Chem Toxicol 2008; 46: $1184-1189$

19 Rhee SJ, Lee JE, Lee CH. Importance of lactic acid bacteria in Asian fermented foods. Microb Cell Fact 2011; 10 (Suppl 1): S5.

20 Hsieh YH, Ofori JA. Innovations in food technology for health. Asia Pac J Clin Nutr 2007; 16 Suppl 1: 65-73.

21 Ahmad A, Anjum FM, Zahoor T, Nawaz H, Dilshad SM. Beta glucan: a valuable functional ingredient in foods. Crit Rev Food Sci Nutr 2012; 52: 201-212.

22 Stern R. Hyaluronan and the process of aging in skin. In: Farage M, Miller K, Maibach H, eds. Textbook of Aging Skin, Springer Berlin Heidelberg, 2010; 225-238.

23 Nishifuji K, Yoon JS. The stratum corneum: the rampart of the mammalian body. Vet Dermatol 2013; 24: 60-72.e15-6.

24 Jiang SJ, Chu AW, Lu ZF, Pan MH, Che DF, Zhou XJ. Ultraviolet B-induced alterations of the skin barrier and epidermal calcium gradient. Exp Dermatol 2007; 16: 985-992.

25 Dai G, Freudenberger T, Zipper P, et al. Chronic ultraviolet B irradiation causes loss of hyaluronic acid from mouse dermis because of down-regulation of hyaluronic acid synthases. Am J Pathol 2007; 171: 1451-1461.

26 Averbeck M, Gebhardt CA, Voigt S, et al. Differential regulation of hyaluronan metabolism in the epidermal and dermal compartments of human skin by UVB irradiation. J Invest Dermatol 2007; 127: 687-697.

27 Kakizaki I, Itano N, Kimata K, et al. Up-regulation of hyaluronan synthase genes in cultured human epidermal keratinocytes by UVB irradiation. Arch Biochem Biophys 2008; 471: 85-93. 